\title{
NEAR-INFRARED BRIGHTNESS OF THE GALILEAN SATELLITES ECLIPSED IN JOVIAN SHADOW: A NEW TECHNIQUE TO INVESTIGATE JOVIAN UPPER ATMOSPHERE
}

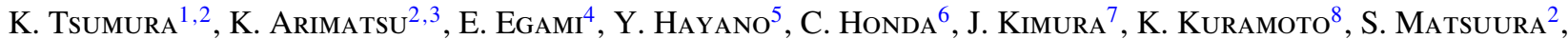 \\ Y. Minowa ${ }^{5}$, K. Nakajima ${ }^{9}$, T. Nakamoto ${ }^{10}$, M. Shirahata $^{2,11}$, J. Surace $^{12}$, Y. Takahashi ${ }^{8}$, And T. Wada $^{2}$ \\ ${ }^{1}$ Frontier Research Institute for Interdisciplinary Science, Tohoku University, Sendai, Miyagi 980-8578, Japan; tsumura@ astr.tohoku.ac.jp \\ 2 Department of Space Astronomy and Astrophysics, Institute of Space and Astronoutical Science, \\ Japan Aerospace Exploration Agency, Sagamihara, Kanagawa 252-5210, Japan \\ ${ }^{3}$ Department of Astronomy, Graduate School of Science, The University of Tokyo, Tokyo 113-0033, Japan \\ ${ }^{4}$ Department of Astronomy, Arizona University, Tucson, AZ 85721, USA \\ ${ }^{5}$ Hawaii Observatory, National Astronomical Observatory of Japan, Hilo, HI 96720, USA \\ ${ }^{6}$ Research Center for Advanced Information Science and Technology, Aizu Research Cluster for Space Science, \\ The University of Aizu, Aizu-Wakamatsu, Fukushima 965-8589, Japan \\ ${ }^{7}$ Earth-Life Science Institute, Tokyo Institute of Technology, Tokyo 152-8550, Japan \\ ${ }^{8}$ Department of Cosmosciences, Graduate School of Science, Hokkaido University, Sapporo, Hokkaido 060-0810, Japan \\ ${ }^{9}$ Department of Earth and Planetary Sciences, Kyushu University, Fukuoka 812-8581, Japan \\ ${ }^{10}$ Department of Earth and Planetary Sciences, Graduate School of Science and Engineering, Tokyo Institute of Technology, Tokyo 152-8551, Japan \\ ${ }^{11}$ National Astronomical Observatory of Japan, Mitaka, Tokyo 181-8588, Japan \\ 12 Spitzer Science Center, California Institute of Technology, Pasadena, CA 91125, USA \\ Received 2014 March 25; accepted 2014 May 20; published 2014 June 20
}

\begin{abstract}
Based on observations from the Hubble Space Telescope and the Subaru Telescope, we have discovered that Europa, Ganymede, and Callisto are bright around $1.5 \mu \mathrm{m}$ even when not directly lit by sunlight. The observations were conducted with non-sidereal tracking on Jupiter outside of the field of view to reduce the stray light subtraction uncertainty due to the close proximity of Jupiter. Their eclipsed luminosity was $10^{-6}-10^{-7}$ of their uneclipsed brightness, which is low enough that this phenomenon has been undiscovered until now. In addition, Europa in eclipse was $<1 / 10$ of the others at $1.5 \mu \mathrm{m}$, a potential clue to the origin of the source of luminosity. Likewise, Ganymede observations were attempted at $3.6 \mu \mathrm{m}$ by the Spitzer Space Telescope, but it was not detected, suggesting a significant wavelength dependence. It is still unknown why they are luminous even when in the Jovian shadow, but forward-scattered sunlight by hazes in the Jovian upper atmosphere is proposed as the most plausible candidate. If this is the case, observations of these Galilean satellites while eclipsed by the Jovian shadow provide us with a new technique to investigate the Jovian atmospheric composition. Investigating the transmission spectrum of Jupiter by this method is important for investigating the atmosphere of extrasolar giant planets by transit spectroscopy.
\end{abstract}

Key words: atmospheric effects - eclipses - planets and satellites: individual (Jupiter, Europa, Ganymede, Callisto)

Online-only material: color figures

\section{INTRODUCTION}

The Galilean satellites (Io, Europa, Ganymede, and Callisto) around Jupiter undergo frequent eclipses in the Jovian shadow. The uneclipsed luminosity of the Galilean satellites is dominated by reflected sunlight at optical and near-infrared wavelengths. The intrinsic blackbody emission from their $120 \mathrm{~K}$ surfaces is negligible, and so they are expected to be truly dark during eclipse. The only exception to this is Io, whose thermal emission from volcanoes can be observed during its eclipse (de Pater et al. 2004). These eclipse events provide us unique opportunities for a variety of studies. The historic first measurement of the speed of light was achieved by observations of eclipses of Galilean satellites (Römer 1676), and this kind of observation has recently been used to obtain accurate astrometric data with great precision (Emelyanov \& Gilbert 2006; Emelyanov 2009; Mallama et al. 2010; Emelyanov et al. 2011). The Jovian upper atmosphere was previously investigated through satellite eclipse events (Smith et al. 1977; Greene et al. 1980; Smith 1980; Smith \& Greene 1980), which allowed studies of the Jovian atmosphere through Earth-based astronomical observations as opposed to in situ studies by spacecraft missions to Jupiter. These eclipse studies were based on the shape of the ingress and egress light curves (Mallama 1991, 1992), since no emission was expected to be detectable during the eclipse itself.
Here, we report unexpected detections of Europa, Ganymede, and Callisto eclipsed in the Jovian shadow at around $1.5 \mu \mathrm{m}$ using the Hubble Space Telescope and the Subaru Telescope. Our motivation in observing the Galilean satellites in eclipse by such great telescopes is to detect the extragalactic background light in the near-infrared wavelengths (Cambresy et al. 2001; Matsumoto et al. 2005; Tsumura et al. 2013) without any zodiacal light subtraction uncertainty by using Galilean satellites eclipsed by Jupiter as occulting spots. Thus, we expected that the Galilean satellites in eclipse are dark enough as occulters in the near-infrared wavelengths, but we found they are bright even in the Jovian shadow. The source of the brightness of these satellites in eclipse remains inconclusive, although some candidates are discussed in this paper, which is why this new finding has the potential to reveal new insights about Jupiter and/or Galilean satellites. For example, in this paper and T. Nakamoto et al. (2014, in preparation), forward-scattered sunlight by hazes in the Jovian upper atmosphere is suggested to be the most probable illuminator. If this is the case, by monitoring many eclipses with different configurations, we can investigate the composition of the Jovian atmosphere, especially the abundance of methane and haze particles, as a function of the position (latitude and altitude) of the atmosphere by observations from Earth. We believe that this method makes it possible to estimate the information of haze particles around the top of the 
Table 1

Our Observations of Eclipses

\begin{tabular}{|c|c|c|c|c|c|c|}
\hline Satellite & $\begin{array}{l}\text { Date } \\
\text { (UT) }\end{array}$ & Telescope & Instrument & Filter & Impact parameter & Brightness \\
\hline Europa & 2012 Feb 21 & Subaru & IRCS & $J$-band $(1.25 \mu \mathrm{m})$ & $0.57-0.62$ & $<1.5 \mu \mathrm{Jy}$ \\
\hline Europa & 2013 Apr 8 & Hubble & WFC3 & $\mathrm{F} 139 \mathrm{M}(1.39 \mu \mathrm{m})$ & $0.54-0.76$ & $<5.5 \mu \mathrm{Jy}$ \\
\hline Europa & 2013 Nov 18 & Subaru & IRCS & $\mathrm{CH}_{4}$-long $(1.69 \mu \mathrm{m})$ & $0.76-0.85$ & $<88 \mu \mathrm{Jy}^{\mathrm{a}}$ \\
\hline Europa & 2014 Mar 19 & Subaru & IRCS & $\mathrm{CH}_{4}$-long $(1.69 \mu \mathrm{m})$ & $0.32-0.96$ & $<0.21 \mu \mathrm{Jy}$ \\
\hline Europa & 2014 Mar 26 & Hubble & WFC3 & $\mathrm{F} 139 \mathrm{M}(1.39 \mu \mathrm{m})$ & $0.30-0.65$ & $6.0-9.5 \mu \mathrm{Jy}$ \\
\hline Ganymede & $2012 \operatorname{Mar} 26$ & Spitzer & IRAC & Channel $1(3.6 \mu \mathrm{m})$ & $>0.87$ & $<3.6 \mu \mathrm{Jy}$ \\
\hline Ganymede & 2012 Jul 26 & Subaru & IRCS & $J$-band $(1.25 \mu \mathrm{m})$ & $0.86-0.95$ & $60-100 \mu \mathrm{Jy}$ \\
\hline Ganymede & 2013 Feb 5 & Hubble & WFC3 & F160W $(1.60 \mu \mathrm{m})$ & $0.77-0.94$ & $60-80 \mu \mathrm{Jy}$ \\
\hline Ganymede & 2013 Mar 5 & Hubble & WFC3 & $\mathrm{F} 139 \mathrm{M}(1.39 \mu \mathrm{m})$ & $0.79-0.74$ & $25-35 \mu \mathrm{Jy}$ \\
\hline Callisto & 2013 Oct 20 & Subaru & IRCS & $J$-band $(1.25 \mu \mathrm{m})$ & $0.88-0.94$ & $20-40 \mu \mathrm{Jy}$ \\
\hline
\end{tabular}

Note. ${ }^{\mathrm{a}}$ Bad weather

stratosphere of the Jovian atmosphere, where the clouds and hazes are mainly produced by photochemistry and play important roles in the cloud formation as condensation nuclei, but are poorly known. Since their brightness in eclipse was $10^{-6}-10^{-7}$ of their brightness out of eclipse, this phenomenon has been undiscovered until now and the state-of-the-art telescopes are required to detected such dark signals in the limited duration time of eclipses.

\section{METHOD}

\subsection{Data Acquisition}

Our observations were conducted with the Infrared Camera and Spectrograph (IRCS) (Kobayashi et al. 2000) using adaptive optics (AO188; Hayano et al. 2010) with the natural guide star mode on the Subaru telescope (Iye et al. 2004), the Wide Field Camera 3 (WFC3; MacKenty 2012) on the Hubble Space Telescope, and the Infrared Array Camera (IRAC; Fazio et al. 2004) on the Spitzer Space Telescope (Werner et al. 2004). Our observations are summarized in Table 1 . Since our observational targets are specific astronomical events, the opportunities for observation are limited and timing is absolutely critical. The separation from the Jovian limb to the satellites is less than 1 arcmin in our observations, thus the greatest difficulty results from stray light due to the close proximity of Jupiter to the target satellites. Therefore, we developed an advanced method for our observations. Although details of our method depend on each observation, a basic strategy is as follows. Jupiter was kept out of the detector field of view (FoV) during the observation to avoid electric crosstalk on the detector array and stray light from Jupiter, which is $\sim 10^{8}$ brighter than the eclipsed satellites. Observations were conducted with non-sidereal tracking on Jupiter (outside of the FoV) to fix the stray light pattern on the detector during the observation. ${ }^{13}$ This minimizes the systematic error due to the Jovian stray light. Owing to absorption by methane in the Jovian atmosphere, Jupiter is dark in methane bands $\left(\mathrm{CH}_{4}\right.$-long filter for Subaru/IRCS, F139M for Hubble/ WFC3, and Channel 1 for Spitzer/IRAC), so observations in the methane bands are very effective for reducing the stray light from Jupiter. Short integration times were required to avoid smearing of the target satellite owing to the relative movement of the target satellites to Jupiter. Allowing the eclipsed satellite

\footnotetext{
13 Only the observation of the Ganymede eclipse by Hubble on 2013 February 5 was conducted with tracking on Ganymede.
}

move relative to the detector using Jupiter tracking has the advantage of effectively dithering the observations so that we can average out any detector issues. The satellite ephemerides are provided with great precision by the Natural Satellites Ephemeride Server MULTI-SAT ${ }^{14}$ (Emel'Yanov \& Arlot 2008) and the JPL-HORIZONS ${ }^{15}$ (Giorgini et al. 1996). The size and location of the eclipsed satellite are accurately known and are therefore easily differentiated from detector effects.

\subsection{Data Processing}

In addition to the standard data reduction such as dark frame subtraction, flat-field correction, and calibration using standard stars, we conducted a special data processing described below.

In each observation we obtained a time series of the observed images, in which the target satellite in eclipse was moving in the fixed stray light pattern from Jupiter outside of FoV. The stray light pattern for each single frame was constructed from other images in which the target satellites moved more than the satellite size (0.8-1.6 arcsec) from the single frame, and was subtracted. In the case of observations with the methane bands, the stray light level from Jupiter is equal to or less than the sky level, so the uncertainty due to the stray light subtraction is also equal to or less than the uncertainty from the photon noise from the sky level. In the case of observations with non-methane bands ( $J$-band filter for Subaru/IRCS and F160W for Hubble/ WFC3), the stray light level from Jupiter was 5 to 12 times brighter than the sky level, so the uncertainty from the photon noise from the stray light was added to that from the sky level. However, the stray light pattern from Jupiter was successfully canceled out due to the observations with Jupiter tracking; thus, systematic uncertainty from the stray light subtraction should be negligible. Another Galilean satellite out of eclipse $\left(\sim 10^{6}\right.$ brighter than the eclipsed satellites) was in the images in some data (Subaru/IRCS data in 2012 February 21 and 2012 July 26, and Hubble/WFC3 data in 2013 February 5 and 2013 April 8), and the target satellite in eclipse was contaminated by the nearby bright satellite. Since the satellites of the contamination source had different movements relative to the target satellite in eclipse and Jupiter, a contamination pattern was not fixed in the images. Even in such a case, since the contamination pattern does not

\footnotetext{
${ }^{14}$ http://www.sai.msu.ru/neb/nss/nssphe0he.htm

$15 \mathrm{http}: / /$ ssd.jpl.nasa.gov/horizons.cgi
} 

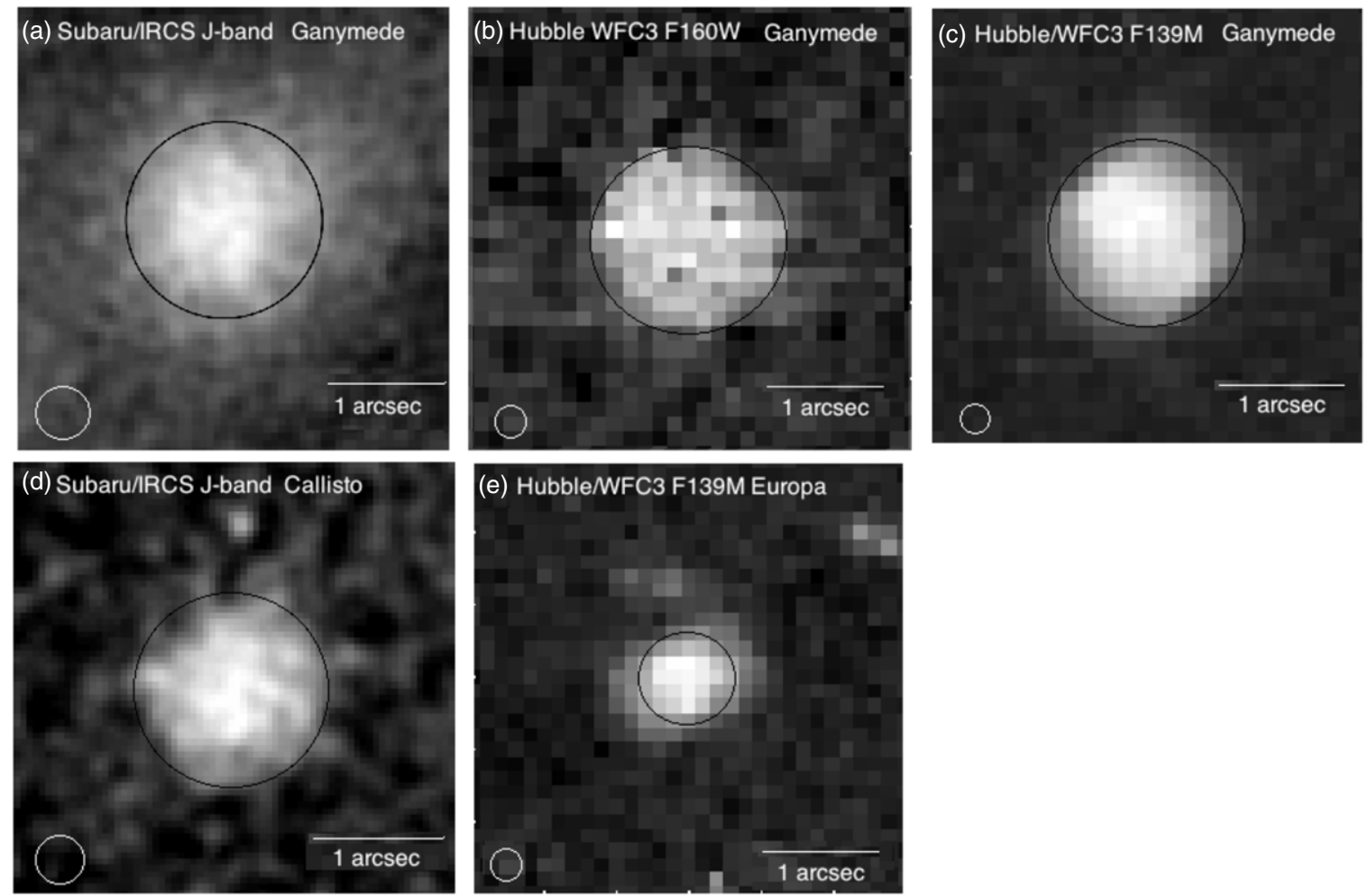

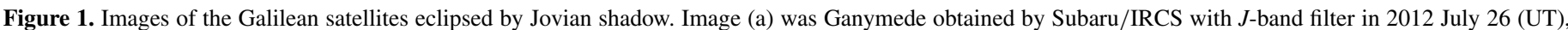

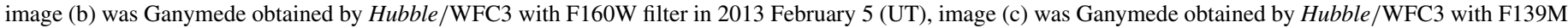

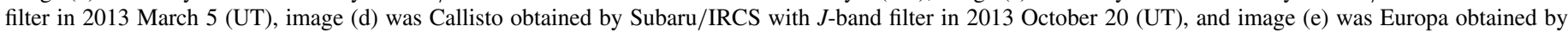

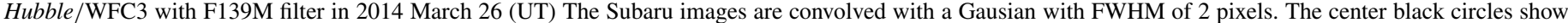
the size of Ganymede or Callisto, and the small white circles at the bottom left corner show the PSF size (FWHM) in each image.

have a local structure, the contamination pattern was evaluated around the target satellite and subtracted locally. ${ }^{16}$

After the stray light subtraction, brightness of the target satellite in eclipse was evaluated. Since we know the exact position and diameter of the satellites, and we can correct the positioning information in the FITS header of each image by comparing it to field stars and the satellite out of eclipse in the images, we can find the pixels where the target satellite in eclipse should exist even in the case of non-detection. The amount of positioning correction by comparing to field stars was less than five pixels. The total uncertainty after the subtraction of the stray light from Jupiter and the satellite out of eclipse was evaluated from the deviation of the pixels around the target satellite in eclipse.

\section{RESULT}

The brightness of the Ganymede in eclipse was $\sim 80 \mu \mathrm{Jy}$ ( $\sim 19.1 \mathrm{AB}$ mag) in the $J$ band, which was $\sim 4 \times 10^{-6}$ extinction from the brightness of Ganymede out of eclipse, and the brightness of the Callisto in eclipse was $\sim 30 \mu \mathrm{Jy}(\sim 20.2 \mathrm{AB}$ mag) in the $J$ band, which was $\sim 2 \times 10^{-6}$ extinction from the brightness of Callisto out of eclipse. On the other hand, Ganymede in eclipse was not detected at $3.6 \mu \mathrm{m}$ by Spitzer/IRAC, obtaining an upper limit of its brightness as $<3.6 \mu \mathrm{Jy}$ ( $>22.5 \mathrm{AB}$ mag). Europa in eclipse was also observed around $1.5 \mu \mathrm{m}$, and the

\footnotetext{
16 Since the observation of the Ganymede eclipse by Hubble on 2013 February 5 was conducted with tracking on Ganymede, the stray light subtraction using the fixed pattern was not applied. However, since Europa out of eclipse was located by Ganymede in eclipse, uncertainty by the contamination subtraction from Europa dominated this data.
}

brightness of Europa was much darker than Ganymede and Callisto. Europa in eclipse was not detected by observations until 2014 March 19, obtaining an upper limit of $<5.5 \mu \mathrm{Jy}(>22.0$ $\mathrm{AB}$ mag) with F139M filter and $<0.21 \mu \mathrm{Jy}$ ( $>25.6 \mathrm{AB}$ mag) with $\mathrm{CH}_{4}$-long filter, but it was detected on 2014 March $26 \mathrm{ob}$ taining $\sim 7 \mu \mathrm{Jy}(\sim 21.8 \mathrm{AB}$ mag) with the same F139M filter. This discrepancy is discussed in Section 4.3. Note that the nondetection of Europa in eclipse at $<820 \mathrm{~nm}$ by Hubble/WFC2 was also reported (Sparks et al. 2010).

Figure 1 shows the images of these detected satellites in eclipse. Since the satellites moved in the images in the time series data, images in Figure 1 were obtained by shift-and-add of the time series images. The sky surrounding the target satellites in Figure 1 has no structure, which shows that the stray light was successfully subtracted. Due to the fine spatial resolutions of Hubble/WFC3 and Subaru/IRCS+AO188, images of these satellites were spread over several pixels as shown in Figure 1, and we found that they were uniformly bright in eclipse. Figure 2 shows the variance of brightness of the satellites in eclipse as a function of impact parameter ${ }^{17}$ from the time series data, and small dependences on impact parameter were detected within the shallow eclipse range (impact parameter $>0.7$ ). Subaru data had a larger dispersion than Hubble data because of the instability of Earth's atmosphere. Figure 3 shows the broadband spectral energy distribution (SED) of Ganymede in eclipse. Note that relative relation among these data points in Figure 3 should not be accepted at face value because each data point was obtained on different days; Thus, the geometric relation among Jupiter, Ganymede, and the observer is different.

\footnotetext{
17 Impact parameter is defined as the ratio of the distance of the satellite from the center of the shadow to the Jovian radius along the target satellite.
} 


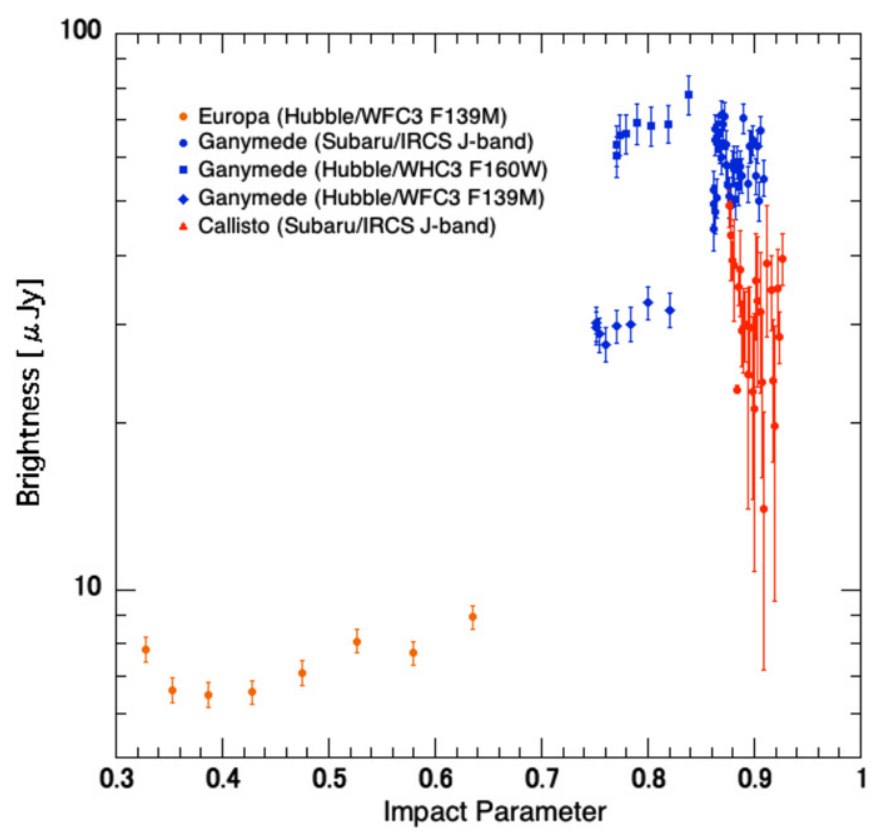

Figure 2. Brightness variance of observed eclipses as a function of impact parameter. Orange points indicate Europa obtained, blue points indicate Ganymede (different symbols show different observations), and red points indicate Callisto. (A color version of this figure is available in the online journal.)

\section{DISCUSSION}

Why are the Galilean satellites bright in the Jovian shadow? We consider the following as possible explanations. (1) Atmospheric emission from the satellites, (2) illumination by emission from the dark side of Jupiter, (3) illumination by sunlight reflected from the other satellites, and (4) illumination by forward-scattered sunlight at the Jovian upper atmosphere to the satellites.

\subsection{Atmospheric Emission from the Satellites}

One possible explanation is (1) the atmospheric emission from the satellites in eclipse. Auroral emission from electron excited atomic oxygen at UV (130.4 and $135.6 \mathrm{~nm}$ ) (Hall et al. 1998; McGrath et al. 2013) and optical (630.0 and $636.3 \mathrm{~nm})$ (Brown \& Bouchez 1999) at both poles of Ganymede in eclipse was known, but this auroral emission cannot explain the uniformity of brightness as shown in Figure 1. Thus, the origin of this brightness should be radiation from whole atmosphere of the satellites. In the case of the Earth atmosphere, $\mathrm{OH}$ molecules are the main carrier of the airglow emission in the near-infrared wavelengths, which are excited by sunlight at dayside and emit photons at nightside. The Galilean satellites are also expected to have $\mathrm{OH}$ molecules in their atmosphere, since $\mathrm{OH}$ molecules can be generated from water ice on their surfaces by sputtering due to interaction with the plasma particles around Jupiter. Such $\mathrm{OH}$ molecules in their atmosphere can be excited by the sunlight while out of eclipse and emit photons during their eclipses. The column density of $\mathrm{OH}$ is estimated to be $10^{10}-10^{11}$ $\mathrm{cm}^{-2}$ in the Ganymede atmosphere (Marconi 2007), which is comparable to that of the $\mathrm{OH}$ layer in the Earths atmosphere. However, the Ganymede SED, especially the non-detection at $3.6 \mu \mathrm{m}$, is difficult to explain by $\mathrm{OH}$ airglow because there is an emission band $(\Delta v=1)$ at 2.5-3.5 $\mu \mathrm{m}$ (Stair et al. 1985) as shown in Figure 3, although the excitation state of $\mathrm{OH}$ molecules and chemical environment in the atmosphere

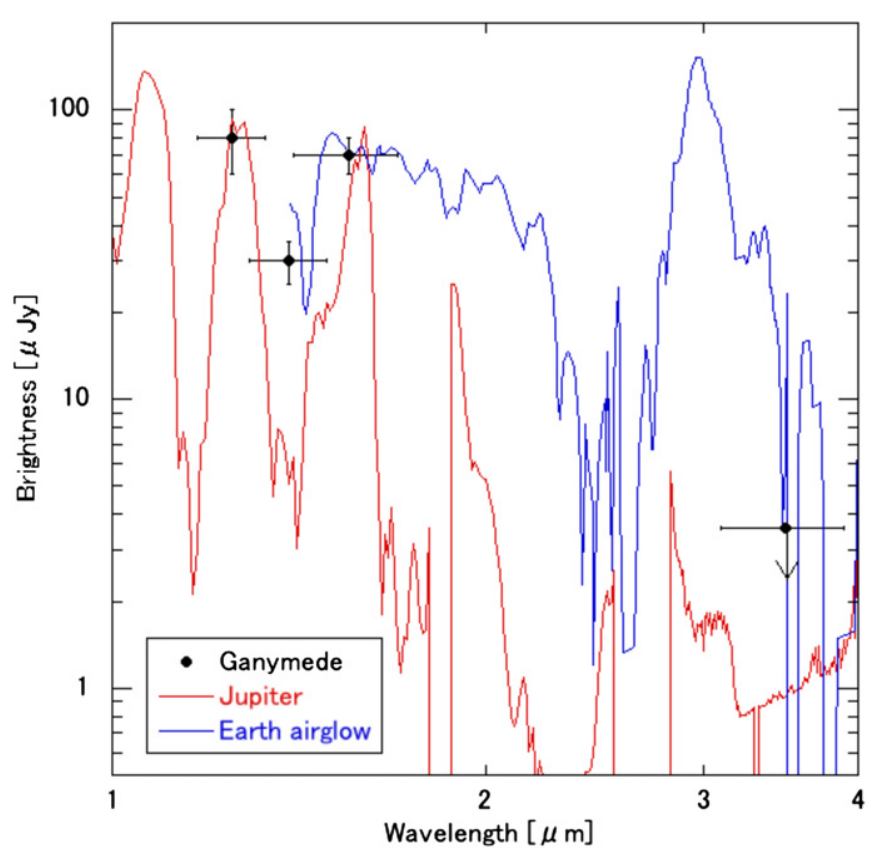

Figure 3. Spectral energy distribution (SED) of Ganymede in eclipse. Each data point of Ganymede in eclipse was obtained in the different date and impact parameter, thus the geometric relation among Jupiter, Ganymede, and the observer is different. Spectra of Jupiter (Rayner et al. 2009) and the Earths atmosphere (Stair et al. 1985) are also shown as a reference, scaled to the Ganymede brightness.

(A color version of this figure is available in the online journal.)

of the Earth and the Galilean satellites are different, resulting in different spectral shapes. The density and composition of Europa's atmosphere are very similar to those of Ganymede and Callisto, therefore it is difficult to explain the large difference in observed brightness among the three. Additionally, atmospheric emission was detected from Europa by the Cassini spacecraft during a fly-by, but not from Ganymede (Porco et al. 2003), which is inconsistent with this explanation.

\subsection{Emission from the Dark Side of Jupiter}

The second candidate is (2) illumination by emission from the dark side of Jupiter. Lightning (Dyudina et al. 2004), aurora (Kim et al. 1993; Gladstone et al. 2007; Radioti et al. 2011), and nightside airglow (Gladstone et al. 2007) of Jupiter are known and they can be candidates of the illuminator. However, it is difficult for these illuminators to explain the fact that the brightness of Europa is much darker than that of Ganymede and Callisto because Europa is located closer to Jupiter, thus Europa should be illuminated more strongly from Jupiter than the others. In addition, the brightness of the satellites in eclipse was kept during our observations (more than one hour in some observations), thus it is also difficult to be explained by sporadic events like lightning and aurora.

\subsection{Sunlight Reflected from the Other Satellites}

We estimated the effect of (3) illumination by the reflected sunlight from the other satellites. Assuming that Io out of eclipse as an illuminator is located $3 \times 10^{5} \mathrm{~km}$ away from Europa in eclipse, ${ }^{18}$ the brightness of Io seen from Europa (with a

\footnotetext{
18 Orbital radii from Jupiter are $4.2 \times 10^{5} \mathrm{~km}$ for Io, $6.7 \times 10^{5} \mathrm{~km}$ for Europa, $1.1 \times 10^{6} \mathrm{~km}$ for Ganymede, and $1.9 \times 10^{6} \mathrm{~km}$ of Callisto. Thus, the assumed distance of $3 \times 10^{5} \mathrm{~km}$ is almost the closest case.
} 
phase angle of $0^{\circ}$ ) is $\sim 6 \times 10^{6}$ of that seen from the Earth. Since extinction owing to phase angle (Sun-Io-Europa) is $>10^{2}$ when the phase angle is $>135^{\circ 19}$ (Simonelli \& Veverka 1984), the brightness of Io seen from Europa with the phase angle of $135^{\circ}$ becomes $\sim 6 \times 10^{4}$ of that seen from the Earth. In addition, the brightness of the Sun seen from Jupiter (or Io/Europa) is $\sim 4 \times 10^{-2}$ of that seen from the Earth. We know that the brightness ratio between the Sun and Io seen from the Earth is $\sim 1 \times 10^{13}$, then we obtained their brightness ratio seen from Europa as $<10^{7}$.

This effect cannot explain the detected brightness of Ganymede and Callisto, whose brightness was $\sim 10^{-6}$ of that out of eclipse, but it can explain the detected brightness of Europa, whose brightness was $\sim 10^{-7}$ of that out of eclipse. In fact, when we detected the Europa brightness in eclipse on 2014 March 26, the phase angle was $134^{\circ}$ and the distance between Europa and Io was $3.4 \times 10^{5} \mathrm{~km}$. These values are almost same as the assumed values in the estimation, meaning that Europa in eclipse was illuminated from Io in the most efficient configuration on this day. On the other hand, Europa in eclipse was not detected with the same filter on 2013 April 8. On this day, the distance between Europa and Io was $2.8 \times 10^{5} \mathrm{~km}$ and the phase angle was $155^{\circ}$. Io appears brighter by a factor of $\sim 1.5$ by the distance effect, but darker by a factor of $>6$ by the phase angle effect (Simonelli \& Veverka 1984) than that on 2014 March 26. In the same way, the non-detections of Europa on the other days can be explained by the configuration of the Jovian system. Thus, illumination by the reflected sunlight from the other satellites explains both the brightness of Europa on 2014 March 26 and the non-detection in the other days.

\subsection{Forward-scattered Sunlight at Jovian Upper Atmosphere}

We propose a hypothesis that (4) the Galilean satellites are illuminated by sunlight forward-scattered by hazes in the Jovian upper atmosphere to explain the brightness of Ganymede and Callisto in eclipse. A detailed discussion for this model is found in T. Nakamoto et al. (2014, in preparation), which details that illumination is dominated by hazes around the top of the stratosphere of the Jovian atmosphere. A methane absorptionlike feature at $1.4 \mu \mathrm{m}$ is found in the Ganymede SED in eclipse, as in the Jovian spectrum (Rayner et al. 2009) as shown in Figure 3. A methane abundance around the top of the stratosphere is almost same as that at higher pressure regions in the Jovian atmosphere $\left(\sim 10^{-3}\right.$ in mole fraction) (Moses et al. 2005), which can explain the $1.4 \mu \mathrm{m}$ methane-like absorption feature in the Ganymede SED in eclipse if this model is true. The albedo of Ganymede at $>3 \mu \mathrm{m}$ is much smaller than that around $1.5 \mu \mathrm{m}$ due to water ice on its surface (Calvin et al. 1995), which also explains the non-detection of Ganymede in eclipse at $3.6 \mu \mathrm{m}$. In addition, the fact that Europa in eclipse was much darker than the others can be explained by this model because the effect of the scattered sunlight should be less at a satellite closer to Jupiter, and the depth of the observed Europa eclipses were deeper (impact parameters were smaller) than those of Ganymede and Callisto. In such a case, however, the extinction of Callisto $\left(\sim 2 \times 10^{-6}\right)$ should be greater than Ganymede $\left(\sim 4 \times 10^{-6}\right)$ because Callisto is located farther from Jupiter than Ganymede, but this was not the case. The difference of albedo and diameter are canceled out in this extinction comparison. This

\footnotetext{
19 The phase angle (Sun-Io-Europa) of $135^{\circ}$ is also the smallest case because we consider the situation of Europa in the Jovian shadow (i.e., the angle of Sun-Jupiter-Europa is $\sim 180^{\circ}$ ).
}

might be explained by the difference of the haze abundance in the Jovian upper atmosphere because the brightness of satellites in eclipse should depend on where (longitude and latitude) the sunlight was scattered in the Jovian atmosphere. The haze abundance in the Jovian atmosphere depends on position (Zhang et al. 2013), and Callisto might be illuminated by sunlight through the haze-rich region of the Jovian upper atmosphere when we observed it. As shown in Figure 2, all Ganymede and Callisto eclipses we observed were shallow (impact parameter $>0.7$ ), thus they may become darker at deeper eclipses in this hypothesis, which will be tested by our future observations.

As the transmission spectrum of the Earth atmosphere was measured from lunar eclipse observation (Pallé et al. 2009; Vidal-Madjar et al. 2010; García Muñoz et al. 2012), spectra of the Galilean satellites in eclipse could be tracers of the Jovian upper atmosphere, especially the abundance of haze and/ or methane along the Jovian altitude, if this model is correct. In particular, secular change and location dependence of the Jovian atmosphere can be monitored by long-term observations of a number of eclipses. For example, observed brightness variances of Ganymede and Callisto shown in Figure 2 might be explained by the variance of the haze abundance in the Jovian atmosphere. Similar studies were conducted to investigate the composition of the Jovian atmosphere using Galilean satellites' eclipses (Smith et al. 1977; Greene et al. 1980; Smith 1980; Smith \& Greene 1980). However, these previous works were based on ingress and egress eclipse light curves that probed reflected/refracted sunlight through regions of the Jovian upper atmosphere (specifically, the bottom of the Jovian stratosphere at pressures of several hundred mbars). This cannot explain the brightness we observe during total umbral eclipses.

To explain this brightness in the total umbral eclipse, T. Nakamoto et al. (2014, in preparation) propose forwardscattered sunlight by hazes in the upper region of the Jovian atmosphere (specifically, the top of the Jovian stratosphere at pressures of several to several tens of mbars). This pressure range has been poorly observed by previous spectral observations and in-situ observations by the Galileo probe. For example, in previous works, Zhang et al. (2013) explicitly stated that 10 mbar is the top of the sensitivity region, and Banfield et al. (1998) stated that the limit is 20 mbar. Our observation has the potential to resolve the vertical structure of hazes well above this pressure level if this model is correct. The Jovian atmosphere of an even higher region $(<1$ mbar) was investigated by observations of field star occultation by Jupiter (Raynaud et al. 2004; Christou et al. 2013), but occultations of field stars by Jupiter are much rarer than the Galilean satellite eclipses. Therefore, observations of the Galilean satellite eclipsed in the Jovian shadow can be a very unique method to investigate the Jovian upper atmosphere if this hypothesis is correct. The clouds and hazes are mainly produced in this pressure range (West 1988; Fortney 2005), and thus constraining for such a pressure range is very important for the understanding of Jovian cloud dynamics.

New insights about the Jovian atmosphere will be extended to the research of exo-planets because the Jovian atmosphere is the base for atmospheric modeling of exo-planets (Seager \& Deming 2010). In particular, since our new technique will provide us the transmission spectrum of Jupiter projected on the Galilean satellites eclipsed in the Jovian shadow as screens, such a transmission spectrum of Jupiter will be applied to the modeling of the atmosphere of extrasolar giant planets by transit spectroscopy (Brown 2001). Transit spectra of 11 exo-planets have been obtained to date (Swain et al. 2014), 
and in these spectra, methane (Swain et al. 2008) and haze (Pont et al. 2008) were first detected in the atmosphere of the hot-Jupiter HD189733b by near-infrared transit spectroscopies. For example, haze abundance is important to estimate the size of the exo-planet from transit observations (de Kok \& Stam 2012). Thus, Jovian transmission spectrum, including methane absorption and haze forward-scattering like our data, will be a standard of modeling and comparison for the characterization of these transit spectra of exo-planets.

\section{SUMMARY}

As seen above, the origin of the brightness of the Galilean satellites in eclipse is still under discussion, but the forwardscattered sunlight model (T. Nakamoto et al. 2014, in preparation) seems to be more plausible. If this is the case, these kinds of observations will provide us with a new observational method to investigate the composition and time variance of the Jovian atmosphere, especially the abundance of methane and haze, as a function of altitude and position in Jupiter. The transmission spectrum of Jupiter by our new method will be applied to the modeling the transit spectrum of exo-planets.

This research is based on observations made with these instruments: the Subaru Telescope, which is operated by the National Astronomical Observatory of Japan and associated with programs S12A-022, S13B-115 and S14A-080; the NASA/ESA Hubble Space Telescope, obtained at the Space Telescope Science Institute (STScI), which is operated by the Association of Universities for Research in Astronomy, Inc. under NASA contract NAS 5-26555, associated with program \#12980; and the Spitzer Space Telescope, which is operated by the Jet Propulsion Laboratory (JPL), California Institute of Technology under a contract with NASA, associated with program \#80235 and \#90143. This work was supported by the Japan Society for the Promotion of Science, KAKENHI (\#24111717, \#26800112), and NASA through a grant from STScI and JPL.

\section{REFERENCES}

Banfield, D., Conrath, B. J., Gierasch, P. J., Nicholson, P. D., \& Matthews, K. 1998, Icar, 134, 11

Brown, M. E., \& Bouchez, A. H. 1999, BAAS, 31, 1183

Brown, T. M. 2001, ApJ, 553, 1006

Calvin, W. M., Clark, R. N., Brown, R. H., \& Spencer, J. R. 2005, JGR, 100, 19041

Cambŕesy, L., Reach, W. T., Beichman, C. A., \& Jarrett, T. H. 2001, ApJ, 555,563

Christou, A. A., Beisker, W., Casas, R., et al. 2013, A\&A, 556, A118 de Kok, R. J., \& Stam, D. M. 2012, Icar, 221, 517

de Pater, I., Marchis, F., Macintosh, B. A., et al. 2004, Icar, 169, 250

Dyudina, U. A., Del Genio, A. D., Ingersoll, A. P., et al. 2004, Icar, 172, 24

Emelyanov, N. V. 2009, MNRAS, 394, 1037

Emelyanov, N. V., Andreev, M. V., Berezhnoi, A. A., et al. 2011, SoSyR, 45,264

Emel'Yanov, N. V., \& Arlot, J. -E. 2008, A\&A, 487, 759

Emelyanov, N. V., \& Gilbert, R. 2006, A\&A, 453, 1141

Fazio, G. G., Hora, J. L., Allen, L. E., et al. 2004, ApJS, 154, 10

Fortney, J. J. 2005, MNRAS, 364, 649

García Muñoz, A., Zapatero Osorio, M. R., Barrena, R., et al. 2012, ApJ, 755,103

Giorgini, J. D., Yeomans, D. K., Chamberlin, A. B., et al. 1996, BAAS, 28, 1158

Gladstone, G. R., Stern, S. A., Slater, D. C., et al. 2007, Sci, 318, 229

Greene, T. F., Smith, D. W., \& Shorthill, R. W. 1980, Icar, 44, 102

Hall, D. T., Feldman, P. D., McGrath, M. A., \& Strobel, D. F. 1998, ApJ, 499,475

Hayano, Y., Takami, H., Oya, S., et al. 2010, Proc. SPIE, 7736, 77360N

Iye, M., Karoji, H., Ando, H., et al. 2004, PASJ, 56, 381

Kim, S. J., Glenar, D. A., Joyce, R. R., \& Kostiuk, T. 1993, Icar, 102, 99

Kobayashi, N., Tokunaga, A. T., Terada, H., et al. 2000, Proc. SPIE, 4008, 1056

MacKenty, J. W. 2012, Proc. SPIE, 8442, 84421 V

Mallama, A. 1991, Icar, 92, 324

Mallama, A. 1992, Icar, 97, 298

Mallama, A., Stockdale, C., Krobuske, B. A., \& Nelson, P. 2010, Icar, 210,346

Marconi, M. L. 2007, Icar, 190, 155

Matsumoto, T., Matsuura, S., Murakami, H., et al. 2005, ApJ, 626, 31

McGrath, M. A., Jia, X., Retherford, K., et al. 2013, JGRA, 118, 2043

Moses, J. I., Fouchet, T., Bézard, B., et al. 2005, JGR, 110, E08001

Pallé, E., Zapatero Osorio, M. R., Barrena, R., Montañés-Rodríguez, P., \& Martín, E. L. 2009, Natur, 459, 814

Pont, F., Knutson, H., Gilliland, R. L., Moutou, C., \& Charbonneau, D. 2008, MNRAS, 385, 109

Porco, C. C., West, R. A., McEwen, A., et al. 2003, Sci, 299, 1541

Radioti, A., Grodent, D., Gèrard, J. -C., et al. 2011, JGR, 116, A03221

Raynaud, E., Matcheva, K., Drossart, P., Roques, F., \& Sicardy, B. 2004, Icar, 168,324

Rayner, J. T., Cushing, M. C., \& Vacca, W. D. 2009, ApJS, 185, 289

Römer, O. 1676, Journal des Sçavans, 4, 233 (This anonymous report was translated into English and published in 1677, Phil. Trans. R. Soc., 12, 893)

Seager, S., \& Deming, D. 2010, ARA\&A, 48, 631

Simonelli, D. P., \& Veverka, J. 1984, Icar, 59, 406

Smith, D. W. 1980, Icar, 44, 116

Smith, D. W., \& Greene, T. F. 1980, Icar, 44, 134

Smith, D. W., Greene, T. F., \& Shorthill, R. W. 1977, Icar, 30, 697

Sparks, W. B., McGrath, M., Hand, K., et al. 2010, IJAsb, 9, 265

Stair, A. T., Jr., Sharma, R. D., Nadile, R. M., Baker, D. J., \& Grieder, W. F. 1985, JGR, 90, 9763

Swain, M. R., Line, M. R., \& Deroo, P. 2014, ApJ, 784, 133

Swain, M. R., Vasisht, G., \& Tinetti, G. 2008, Natur, 452, 329

Tsumura, K., Matsumoto, T., Matsuura, S., Sakon, I., \& Wada, T. 2013, PASJ, 65,121

Vidal-Madjar, A., Arnold, L., Ehrenreich, D., et al. 2010, A\&A, 523, A57

Werner, M. W., Roellig, T. L., Low, F. J., et al. 2004, ApJS, 154, 1

West, R. A. 1988, Icar, 75, 381

Zhang, X., West, R. A., Banfield, D., \& Yung, Y. L. 2013, Icar, 226, 159 